\title{
Deciphering the 3D Structure and Function of Phosphofructokinase from Fission Yeast
}

\author{
Shaun Benjamin, Michael Radermacher and Teresa Ruiz \\ University of Vermont, Dept. of Molecular Physiology and Biophysics, Burlington, VT 05405
}

Glycolysis is an essential process for the energy metabolism of living cells and is tightly regulated during the cell cycle. A main regulatory step is centered around the behavior of phosphofructokinase (PFK), which catalyzes the phosphorylation of fructose-6-phosphate (F6P) to fructose-1,6-bisphosphate and represents the first irreversible step specific for glycolysis. PFK is ubiquitously expressed in all cellular organisms; however, the subunit composition, oligomerization state, and specific kinetic behavior of this enzyme are species and tissue dependent. While in prokaryotes, PFK is a homo-tetramer composed of identical subunits with a molecular mass of $35 \mathrm{kDa}$ each, in the methylotrophic yeast, Pichia pastoris, PFK (PpPFK) is a dodecamer composed of four $\alpha$-, four $\beta$ - and four $\gamma$-subunits [1]. In addition, the eukaryotic enzymes exhibit a much more complex regulatory mechanism than their prokaryotic counterparts with more than 20 allosteric effectors regulating their activity [2].

Yeast PFKs have been widely studied for basic biological reasons and for commercial applications due to their role in fermentation. Insights into the structural/functional mechanism of the yeast enzymes have been obtained mostly by 3D electron microscopy (3DEM). A study by cryo-EM of Saccharomyces cerevisiae PFK (SCPFK) to $10.8 \AA$ resolution permitted to infer the position of the $\mathrm{N}$-terminal domains of the $\alpha$ - and the $\beta$ subunits and the localization of the catalytic binding region [3,4]. In addition, in combination with molecular replacement, it provided initial phases to determine the X-ray structure of a truncated tetramer in the presence of F6P [5]. The 3D structures of the homo-octameric Schizosaccharomyces pombe PFK (SpPFK) determined in the presence of F6P (active state) and in the presence of ATP (inactive state) revealed a similar organization for both, homo- and hetero-octameric PFKs and have manifested a strong correlation between kinetic behavior and structural conformation [6,7]. A recent X-ray study of the whole $P p$ PFK in the inactive state [8] correlates well with other available yeast structures within the bacterial homologous region, however the important $\mathrm{N}$-terminal regions of the subunits show very high disorder and are only partially resolved. There is still a controversy about the location and regulatory role of the N-terminal regions of the PFK subunits.

The $S p$ PFK is an ideal sample to resolve this conundrum since this PFK has a tendency to form larger supra-oligomeric (PFK strings). This might imply that the core of the molecule is structurally similar to the apical domain where the $\mathrm{N}$-terminal region of the PFK molecules has been modeled in PpPFK. We have analyzed preparations of SpPFK in the presence of 3mM F6P (active-state) by cryo-electron microscopy. Data were collected at nominal magnifications of 50k-60k and acceleration voltage of $100 \mathrm{kV}$ to improve contrast (Fig 1a, background). A defocus range between 1.0-3.0 $\mu \mathrm{m}$ was used to obtain good coverage over the full resolution range. More than 6000 images, adjusted to $2.4 \AA$ pixel-size, were manually extracted from the micrographs. 3D reconstructions were calculated using Radon transform algorithms using 3D reference based projection alignment methods. The resolution of the reconstructions was estimated using Fourier shell correlation methods.


$152 \AA$ in width) and can be described as a dimer of tetramers. The analysis of the orientation between the 
tetramers reveals that the rotation angle between them is identical to the one observed for $S c \mathrm{Pfk}\left(75^{\circ}\right)$. Alignment of the SpPFK segmented tetramers has uncovered that the top and the bottom tetramer show the same identical conformation and has determined that this PFK is symmetrical. This effect might be due to the homo-meric nature of the enzyme. We have taken advantage of the SpPFK symmetry and have increased our data set 4-fold, which has significantly improved the signal in the $3.5-1.5 \mathrm{~nm}$ range and the resolution of the structure (Fig. 1b). We are currently using this higher signal to noise ratio 3D structure as the basic framework for our modeling studies not only in the octameric enzyme but also in the larger supra-oligomers.

\section{References:}

[1] S. Benjamin, et al., J. Struct. Biol. 168 (2009), p. 345.

[2] Sols, A., Current Topics in Cellular Regulation 19 (1981), p. 77.

[3] T. Ruiz, G. Kopperschlager, M. Radermacher, J. Struct. Biol. 136 (2001), p. 167.

[4] T. Ruiz et al., J. Struct. Biol. 143 (2003), p. 124.

[5] K. Banaszak et al. J. Mol. Biol. 407 (2011), p. 284.

[6] M. Barcena et al. J. Struct. Biol. 159 (2007), p. 135.

[7] S. Benjamin et al. J. Struct. Biol. 159 (2007), p. 498.

[8] N. Straeter et al. Faseb J. 25 (2010), p. 89.

[9] This work was supported by NIH R01 grant GM069551 (to T.R.), and has benefited from image analysis developments supported by NIH R01 grant GM078202 (to M.R.)
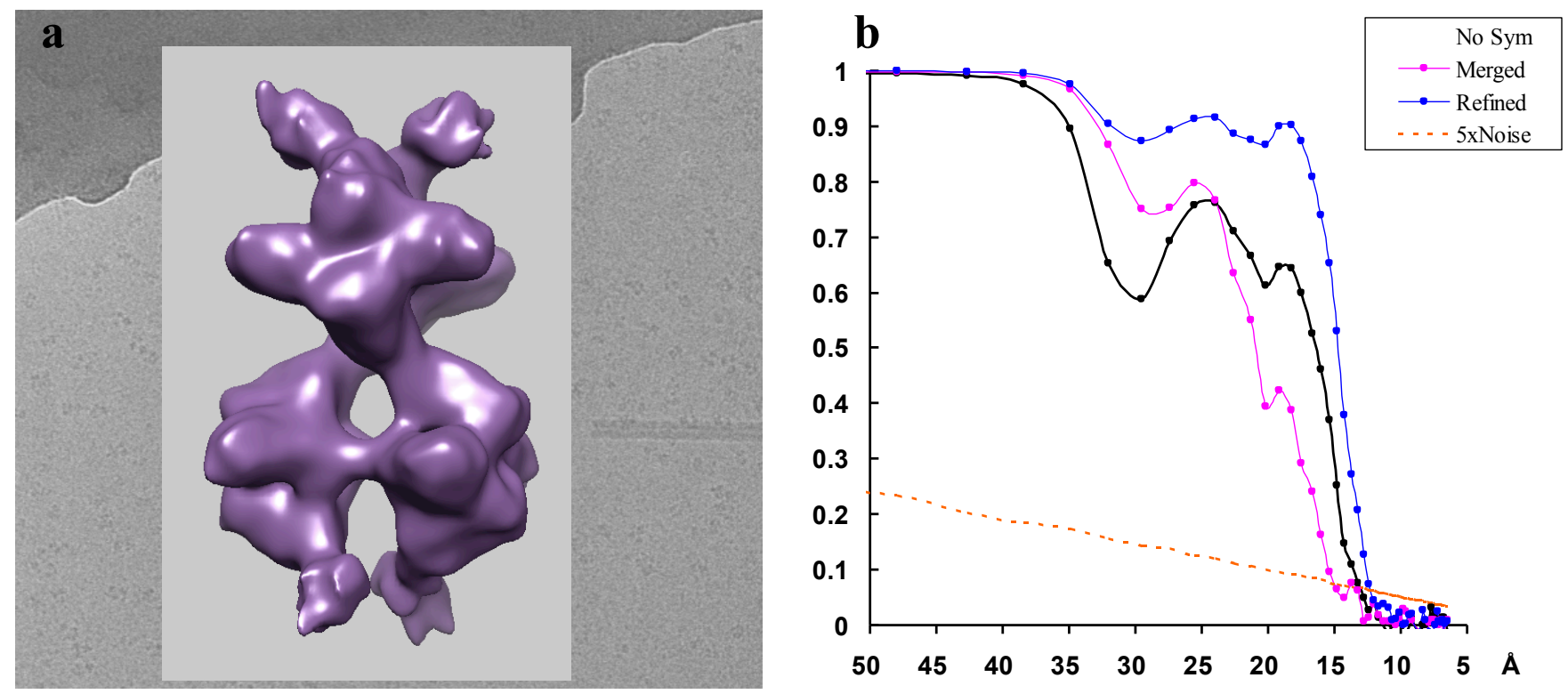

Figure 1. a) Electron micrograph from a cryo-preparation of $S p \mathrm{Pfk}$ in the active state (background), and Cryo-EM 3D structure of the symmetrized SpPfk; (b) FSC resolution curves showing several step of the processing. 